\title{
COSMOLOGICAL PARAMETERS FROM THE X-RAY EVOLUTION OF CLUSTERS
}

\author{
R.G. BOWER AND S.T. KAY \\ University of Durham \\ Department of Physics, Science Laboratories, South Road, \\ Durham DH1 3LE, UK.
}

This poster paper presents an extension of the entropy-driven model of cluster evolution developed by Bower, 1997 (MN, 288, 355) in order to study the evolution of clusters in a low density universe. Our approach allows us to explicitly seperate the contributions from gravitational collapse and changes in the core entropy of the intracluster gas. Here, we apply the model to determine whether a preferred value of $\Omega_{0}$ is selected by currently available constraints (Figure 1). Measurements of the X-ray evolution of clusters cannot, by themselves, select out a particular cosmological model. An additional constraint on the effective power spectrum index $(n)$ is required. This can either come from a measurement of the large-scale galaxy correlation function or from the shape of the present-day temperature function. A theoretical calculation of the $\Omega_{0}$ dependence of the power spectrum does not help break the inherent degeneracy.
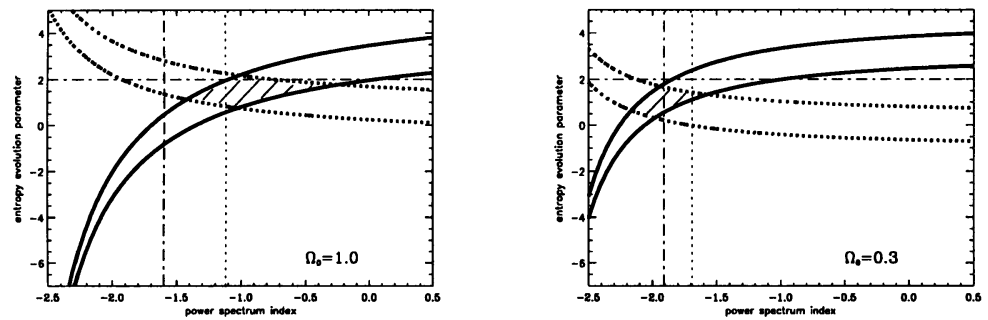

Figure 1. Constraints on values of the spectral index $(n)$ and the entropy evolution parameter $(\epsilon)$ for recent measurements of the X-ray Luminosity Function evolution (thick solid lines) and the X-ray Luminosity-Temperature correlation (thick dotted lines). The shaded region shows values of $n$ and $\epsilon$ satisfying both constraints. Thin vertical lines show CDM predictions of $n$ for $z=0$ (dotted) and $z=0.5$ (dash-dot) in each cosmology. The horizontal lines indicate an upper bound on the value of $\epsilon$. 